\title{
WeChat app-based reinforced education improves the quality of opioid titration treatment of cancer-related pain in outpatients: a randomized control study
}

Zhiyou Peng ${ }^{1+}$, Lin $\mathrm{Li}^{2+}$, Yuan Chen ${ }^{3}$, Zhiying Feng ${ }^{1 *}$ and Xiangming Fang ${ }^{4^{*}}$

\begin{abstract}
Background: As inadequate pain communication contributes to difficulties in optimizing outcomes of outpatients, we investigated the effect of reinforced education using WeChat App to the opioid titration treatment of cancerrelated pain in the outpatient setting.

Methods: We conducted a prospective study to compare reinforced education using Wechat with care as usual from February to December 2019. Patients in the reinforced education group received reinforced education via Wechat, while those in the control group received care as usual. Effect measurements for both groups are carried out with questionnaires at the baseline and 3 days later. Questionnaires include pain intensity (NRS), treatmentrelated adverse events, cancer-related quality of life (QOL), sleep (PSQI), satisfaction, anxiety (GAD-7) and depression (PHQ-9). Number of patients whose NRS reduced to less than three points in $24 \mathrm{~h}$ was the primary outcomes. Secondary outcomes included treatment-related adverse events, cancer-related quality of life, sleep, satisfaction, anxiety and depression.
\end{abstract}

Results: Although there was no significant difference regarding pain intensity (NRS) between the two groups at 72 $h$, the rate of NRS that reduced to less than three points in $24 \mathrm{~h}$ was significantly higher in the Wechat group than in the control group. Patients' satisfaction was significantly higher in the Wechat group than in the control group. There was no significant difference between the two groups regarding the other findings at $72 \mathrm{~h}$, including pain intensity (NRS), cancer-related quality of life (QOL), anxiety (GAD-7), depression (PHQ-9), and sleep (PSQI). However, no significant difference was found between the two groups for constipation, nausea, vomiting, dizziness, somnolence, pruritus, loss of consciousness, and death.

(Continued on next page)

\footnotetext{
* Correspondence: fzy1972@zju.edu.cn; xmfang@zju.edu.cn

†Zhiyou Peng and Lin Li contributed equally to this work.

'Department of Pain Medicine, the First Affiliated Hospital, Zhejiang University School of Medicine, Hangzhou, China

${ }^{4}$ Department of Anesthesiology, the First Affiliated Hospital, Zhejiang University School of Medicine, Hangzhou, China

Full list of author information is available at the end of the article
}

(c) The Author(s). 2020 Open Access This article is licensed under a Creative Commons Attribution 4.0 International License, which permits use, sharing, adaptation, distribution and reproduction in any medium or format, as long as you give appropriate credit to the original author(s) and the source, provide a link to the Creative Commons licence, and indicate if changes were made. The images or other third party material in this article are included in the article's Creative Commons licence, unless indicated otherwise in a credit line to the material. If material is not included in the article's Creative Commons licence and your intended use is not permitted by statutory regulation or exceeds the permitted use, you will need to obtain permission directly from the copyright holder. To view a copy of this licence, visit http://creativecommons.org/licenses/by/4.0/. The Creative Commons Public Domain Dedication waiver (http://creativecommons.org/publicdomain/zero/1.0/) applies to the data made available in this article, unless otherwise stated in a credit line to the data. 
(Continued from previous page)

Conclusions: Our results indicated that receiving instructions delivered by Wechat resulted an increased number of patients with good pain control and better satisfaction. The study provided insight into the effectiveness of the reinforced education using a Wechat app delivered by a doctor to outpatients in the titration treatment of cancerrelated pain.

Trial registration: This study was registered at chictr.org (Registration number: ChiCTR1900021150, Date of Registration: January 30, 2019).

Keywords: WeChat app, Opioid titration treatment, Cancer-related pain, Outpatients

\section{Background}

Pain is the most significant concerns of many patients secondary to their disease and significantly impacting upon their quality of life. It is a considerable challenge to management of this pain successfully, especially for outpatients. Following the World Health Organization (WHO) analgesic ladder, treatment with strong opioids can be considered the mainstay of cancer pain therapy $[1,2]$. However, many terminal cancer patients prefer to be at home than anywhere else. There is an increasing requirement of family carers' role in managing cancerrelated pain and providing palliative care at home [3]. However, cancer patients at home are less likely to have access to adequate analgesia compared to those in hospital $[4,5]$. As pain is a subjective experience, patients who experience pain at home cannot get timely treatment if they cannot recognize and deal with it correctly or cannot contact the doctor in time.

Adequate knowledge and understanding of pain and analgesic medications is critical for managing cancer pain, and influences on the quality of pain management for patients at home [6]. Distinguishing and handling risk factors for adverse reactions, the titration of the dose based on pain level and knowing where to obtain additional information are the foundation of effective cancer pain management. Considering these challenges and the barriers that have been identified in the outpatient setting, patients need to be supported with reinforced education through more comprehensive and timely help $[7,8]$. Thomas ML, et al. promoted education or motivational-interviewing-based coaching compared to usual care to improve cancer pain management [9]. Improving titration treatment of cancer-related pain through traditional communication routines, including face-to-face verbal education, written booklets or visual aids or telephone-based re-instruction, has proven useful [10]. However, the effectiveness and applicability of these interventions have been shown to be inconsistent. So, newly healthcare technology is promising in facilitating telemonitoring to enhance education management.

In recent years, Wechat provided an easily accessible and interactive channel of communication between patients and medical providers, playing an important role in optimizing clinical work $[11,12]$. As a pain monitor has been identified as an important barrier to adequate pain management in the outpatient setting, we tested the hypothesis that reinforced education with Wechat is promising in terms of enhancing the cancer pain management of outpatients.

\section{Methods}

A prospective, randomized, controlled study was conducted at the First Affiliated Hospital, Zhejiang University School of Medicine. The study was approved by the institutional review board of the first affiliated hospital, Zhejiang University School of Medicine, and was registered at chictr.org (ChiCTR1900021150). Written informed consent was obtained from all patients and our study adhered to CONSORT guidelines. According to the WHO's three-step principle, individuals over the age of 18 years of age and not older than 75 years who needs to start opiate titration in outpatient clinics were enrolled in the study, which took place from February to December 2019.

Patients meeting the following inclusion criteria were eligible to participate: subjects voluntarily signed the informed consent for the clinical observation; Subject had a digital score of persistent pain $(\geq 4)$ and (or) number of breakthrough cancer pain ( $\geq 3$ times per day) originated from cancer, regardless of distant metastasis; pain is mainly caused by physical factors, not mental and psychological factors; subjects or caregivers can use Wechat skillfully. We defined breakthrough cancer pain as "a transient exacerbation of pain that occurs either spontaneously, or in relation to a specific predictable or unpredictable trigger, despite relatively stable and adequately controlled background pain" [13]. Patients were excluded if they met the following exclusion criteria: had a history of alcohol abuse, drug or opium abuse; new anti-cancer drugs or new radiotherapy schemes were used during clinical observation; cognitive deficits or mental disorders, or consciousness disorders; pregnancy or lactation; morphine-related contraindications; patients with unstable vital signs; patients participating in another interventional clinical study. 
According to the order of the patients enrolled, the patients were assigned to the Wechat group or the control group according to the computer-generated random numbers plan.

\section{Protocol of opioid titration treatment}

The National Comprehensive Cancer Network's (NCCN) clinical practice guidelines in adult cancer pain, this protocol defines the dose of oral morphine, the interval, absence of limitation of the total dose, numerical rating scale (NRS) threshold required to administer morphine, criteria to stop titration, and how to convert immediate-release morphine into sustained-release oxycodone. Figure 1 describes the titration schedule. Morphine titration is administered until a NRS $\leq 3$ is reached, or until the onset of a serious adverse events. Patients themselves recorded the pain score and adverse events in their record book. Patients were told to go to nearby medical institutions to seek help if they experienced adverse events. Patients considered opioid tolerant were those who had been taking for a week or longer at least $60 \mathrm{mg}$ of morphine daily, or at least $30 \mathrm{mg}$ of oral oxycodone daily, or an equianalgesic dose of another opioid. Pain scores was evaluated every $4 \mathrm{~h}$ and morphine immediate-release tablets were given when the NRS score was greater than 3 . The sustained dose was half of the total amount of morphine immediate-release tablets and oxycodone sustained-release tablets in the first $24 \mathrm{~h}$. The rescue dose is $10-20 \%$ of the total dose of drugs in the first $24 \mathrm{~h}[14,15]$.

\section{Education}

Any questions were answered at the initial appointment. Reinforced education was provided by one experienced doctor through Wechat. Based on the standardized management of cancer pain in the control group, Wechat was used to provide reinforced education. Wechat Subscription Platform was used to publish information including the pain score, knowledge related to opioid analgesics, role of such drugs, common errors when taking tablets, possible adverse reactions, and prevention and treatment methods. Doctors actively and timeously answered patients' concerns regarding the treatment, and marked the department contact telephone on the subscription plat information column. Members of the pain control group used Wechat to communicate using either voice or text depending on the patient's needs in the micro-letter. We tried to enable all participants to achieve satisfactory pain control and handle risk factors related to opioid use.

Other than answering questions at the initial appointment, patients in the control group received no further

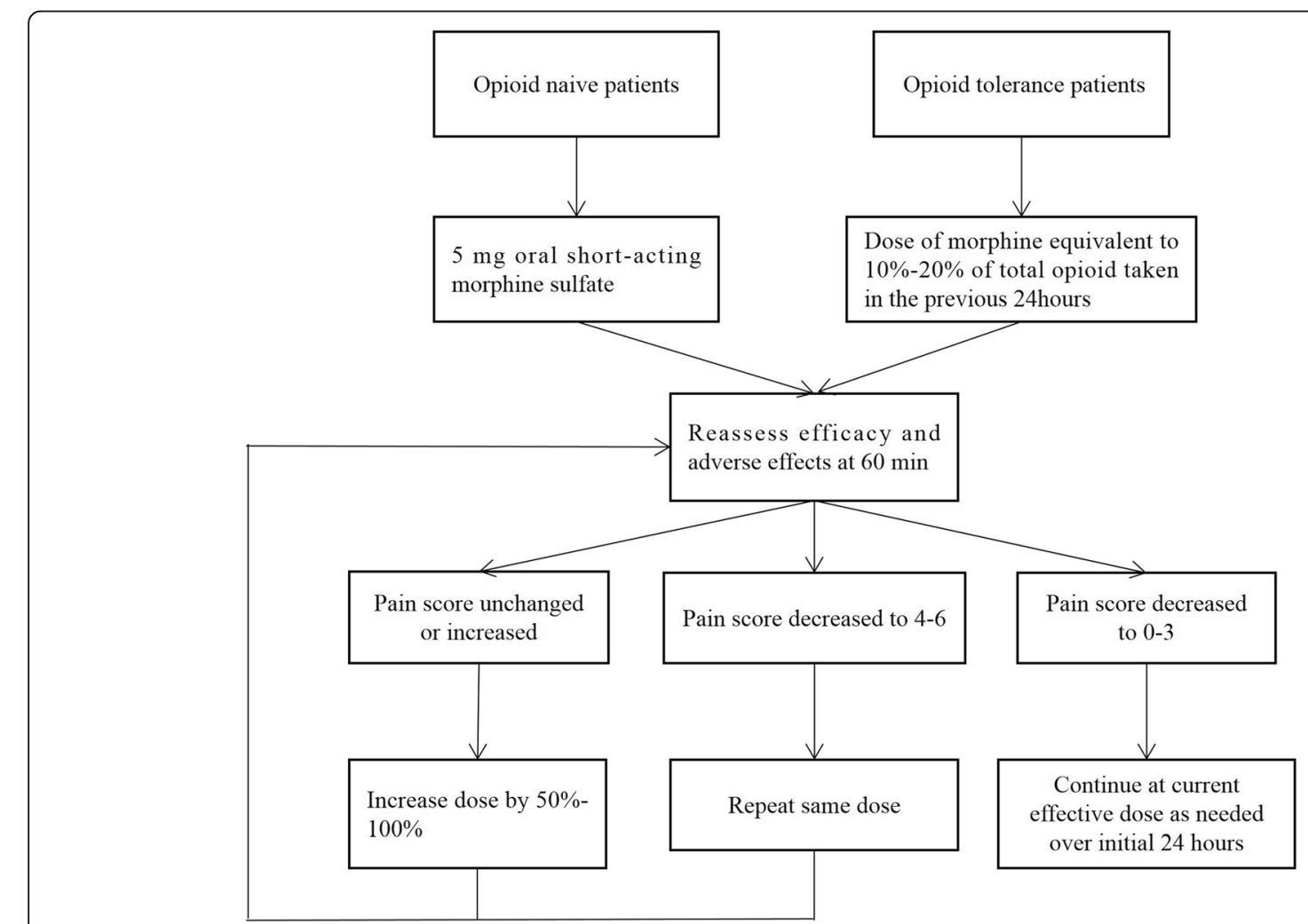

Fig. 1 The schedule of opioid titration treatment 
active education from doctors. Afterward, patients were seen again at the outpatient clinic for further education or contacted by phone for a follow-up consultation. The timing and frequency of the follow-up consultation differed according to patients' general conditions.

Patients in the reinforced education group received reinforced education via Wechat, while those in the control group received care as usual. Wechat Subscription Plat is used to publish information, which includes pain score and opioid analgesics related knowledge. Doctors actively answered patients' doubts in the treatment in time. We strived to let all participants be able to achieve satisfactory pain control and how to handle risk factors related to opioid using. The intervention period and the control period before study assessments were conducted was $72 \mathrm{~h}$ the initial appointment.

\section{Outcome assessments}

Number of patients whose NRS reduced to less than three points in $24 \mathrm{~h}$ was the primary outcomes. Secondary outcomes included pain intensity (NRS), satisfaction with pain management and pain intensity, sleep (Pittsburgh sleep quality index, PSQI), anxiety (Generalized anxiety disorder-7, GAD-7), depression (Patient health questionnaire-9, PHQ-9), and cancer-related quality of life (QOL) at $72 \mathrm{~h}$ after the initionation of treatment. In addition, treatment-related adverse events including nausea, vomiting, dizziness, somnolence, pruritus, loss of consciousness and death were also recorded.

\section{GAD-7 and PHQ-9}

We used the GAD-7 and PHQ-9 to measure the anxiety and depression. The reliability and validity of GAD-7 and PHQ-9 are well established and they are commonly used as self-report tools to evaluate the severity of anxiety and depression. Patients whose scores are greater than 4 on the GAD-7 or the PHQ9 are considered as suffering from anxiety or depression, respectively $[16,17]$.

\section{PSQI}

The PSQI was used to evaluate patients' sleep quality of patients. This scale consists of 18 self-report items that are divided into 7 dimensions, namely subjective sleep quality, time to fall asleep, sleep duration, sleep efficiency, sleep disturbances, use of sleep medication, and daytime dysfunction. The score on each dimension ranges from 0 to 3 and higher total scores indicate lower sleep quality [18].

\section{QOL}

The QOL was used for evaluating the quality of life of cancer patients. This scale consists of 12 self-report items, including appetite, spirit, sleep, fatigue, pain, family coordination, colleagues coordination, oneself understanding of cancer, attitude to treatment, daily life, side effects of treatment, facial expressions. The score on each dimension ranges from 1 to 5 with the total scores as 60 and higher total scores indicate better quality of life.

\section{Statistical analysis}

Patients in the Wechat and control groups were expected to show a $15 \%$ improvement (from 75 to $90 \%$ ) in the rate of NRS $\leq 3$ within $24 \mathrm{~h}$. Thus, a sample size of 130 patients in each group was required for a statistical power of $80 \%$ at a two-tailed significance level of 0.05 . Assuming a 10\% dropout rate, it was deemed that 145 patients per group would be required to obtain statistically significant results for the primary outcome.

Continuous variables were presented as means \pm standard deviation, and categorical data were shown as numbers and percentages. For different variable types, we employ different approaches to evaluate their differences. For categorical variables, we employ Chi-squared tests to evaluate the variable differences. While for continuous variables, we adopt Student's T test to assess their differences. The statistical significance is measured by $p$ value, and $p<0.05$ indicated statistical significance. All analyses were performed using the SPSS software (Ver. 24.0; IBM Corp., New York, USA).

\section{Result}

Between February and December 2019, 380 outpatients were considered for the purposes of this study. Figure 2 illustrated the patient recruitment flowchart. The baseline characteristics of two groups were similar, including the age, gender, level of education, pain site, and message receiver (refer to Table 1).

Although there was no significant difference regarding pain intensity (NRS) between the two groups at $72 \mathrm{~h}$, the rate of NRS that reduced to less than three points in 24 $\mathrm{h}$ was significantly higher in the Wechat group than in the control group. Patients' satisfaction was significantly higher in the Wechat group than in the control group. There was no significant difference between the two groups regarding the other findings at $72 \mathrm{~h}$, including cancer-related quality of life (QOL), anxiety (GAD-7), depression (PHQ-9), and sleep (PSQI) (all $p>0.05$ ) (refer to Table 2).

However, no significant difference was found between the two groups for constipation, nausea, vomiting, dizziness, somnolence, pruritus, loss of consciousness, and death (refer to Table 3).

\section{Discussions}

The study provides insight into the effectiveness of education reinforced with Wechat delivered by a doctor to 


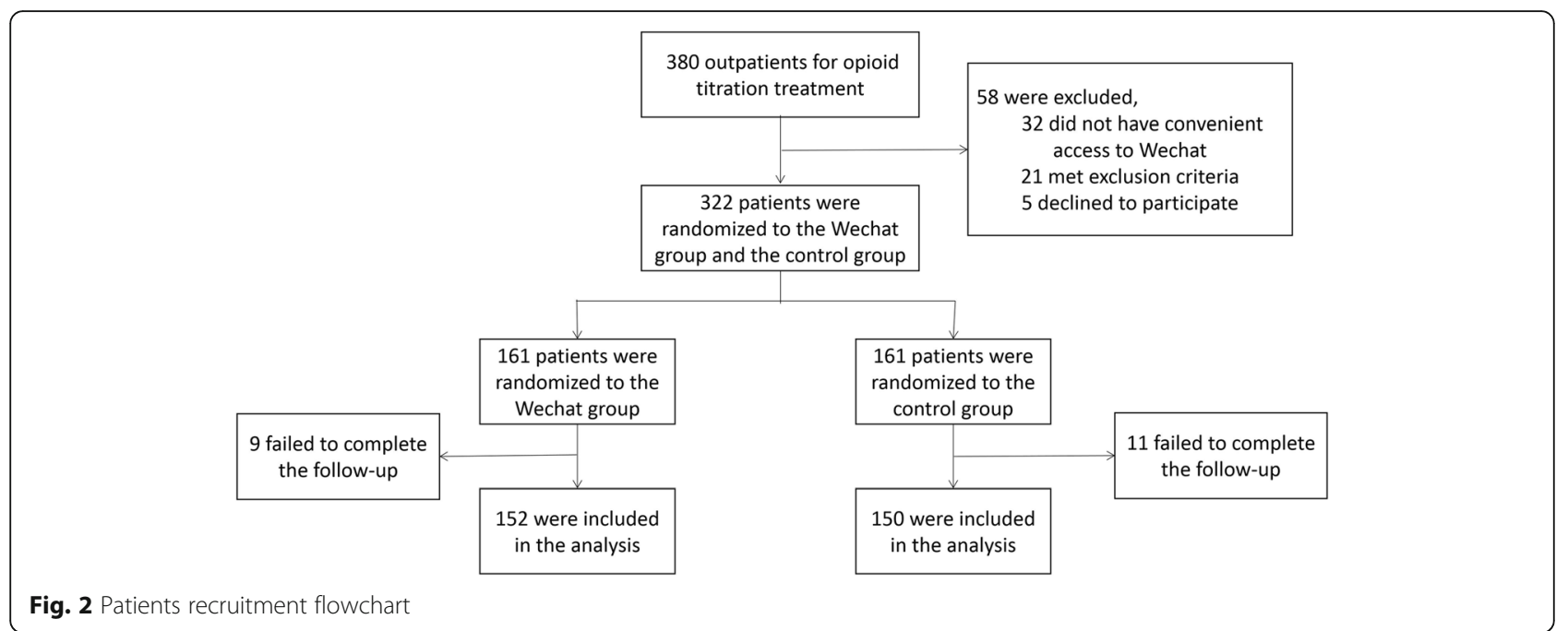

outpatients on the titration treatment of cancer-related pain. Our results indicated that patients receiving instructions delivered by Wechat had a relatively higher quality of opioid titration treatment and better satisfaction.

As actual cancer pain management in outpatient settings have different kinds of problems in practice, clinicians urgently need of a promising solution for follow-ups with outpatients to strengthen pain medication management $[19,20]$. Although information on the use of opioids for improving the opioid titration treatment of cancer-related pain can be easily found on the internet, it's not easy for patients to get what they really need. After evaluated a technology-based multicomponent self-management support intervention that combines the monitoring of pain, adverse effects, and medication with graphical feedback, education, and nurse support, it was found that self-management support results in better pain control and better quality of life than care as usual [21]. The results of a systematic review of intervention studies suggests that educational interventions delivered face-to-face, supported by written and/or other resources, and by appropriate followups can potentially improve family carers' knowledge and self-efficacy for pain management and reduce attitudinal barriers [8]. To the bet of our knowledge, this is the first study demonstrating that Wechat, as an adjunct to regular instructions, improves the opioid titration treatment of cancer-related pain in outpatients. This may be because Wechat allowed patients with more

Table 1 Baseline characteristics of the study patients

\begin{tabular}{|c|c|c|c|}
\hline & & Wechat group $(n=152)$ & Control group $(n=150)$ \\
\hline Age (years), mean (SD) & & $55.6 \pm 6.8$ & $56.3 \pm 7.0$ \\
\hline Female (n) & & $80(52.6 \%)$ & 78 (52.0\%) \\
\hline Smoke history (n) & & $27(17.8 \%)$ & $24(16.0 \%)$ \\
\hline Hypertension (n) & & $33(21.7 \%)$ & $28(18.7 \%)$ \\
\hline Diabetes (n) & & $12(7.9 \%)$ & $14(9.3 \%)$ \\
\hline \multirow[t]{3}{*}{ Level of education (n) } & Elementary school or less & $20(13.2 \%)$ & $22(14.7 \%)$ \\
\hline & High school & $92(60.5 \%)$ & $90(60.0 \%)$ \\
\hline & College or higher & $40(26.3 \%)$ & $38(25.3 \%)$ \\
\hline \multirow[t]{4}{*}{ Pain site (n) } & Abdomen & $92(60.5 \%)$ & $93(62.0 \%)$ \\
\hline & Chest & $38(25 \%)$ & $36(24.0 \%)$ \\
\hline & Limb & $12(7.9 \%)$ & $14(9.3 \%)$ \\
\hline & Head & $10(6.6 \%)$ & $7(4.7 \%)$ \\
\hline \multirow[t]{2}{*}{ Message receiver } & Patients & $32(21.1 \%)$ & $33(22.0 \%)$ \\
\hline & Caregivers & 120 (78.9\%) & 117 (78.0\%) \\
\hline
\end{tabular}


Table 2 Effect of reinforced education with Wechat on the outcome of cancer pain management at $72 \mathrm{~h}$. T0: baseline, T1:72 $\mathrm{h}$ after treatment, ${ }^{*} p<0.05$

\begin{tabular}{|c|c|c|c|c|}
\hline \multirow[t]{2}{*}{ Outcome measures } & \multicolumn{2}{|c|}{ Wechat group $(n=152)$} & \multicolumn{2}{|c|}{ Group Control $(n=150)$} \\
\hline & T0 & $\mathrm{T} 1$ & T0 & $\mathrm{T} 1$ \\
\hline Pain intensity (NRS) & $6.8 \pm 1.9$ & $2.5 \pm 1.2$ & $6.6 \pm 2.1$ & $2.8 \pm 1.1$ \\
\hline Cancer-related quality of life (QOL) & $35.2 \pm 6.5$ & $46.3 \pm 5.5$ & $36.3 \pm 6.2$ & $43.4 \pm 5.2$ \\
\hline Anxiety (GAD-7) & $16.1 \pm 3.3$ & $9.8 \pm 2.2$ & $16.3 \pm 3.6$ & $12.4 \pm 3.1$ \\
\hline Depression (PHQ-9) & $17.2 \pm 3.0$ & $10.1 \pm 2.8$ & $16.7 \pm 3.4$ & $11.2 \pm 2.2$ \\
\hline Sleep (PSQI) & $17.2 \pm 4.2$ & $12.7 \pm 3.3$ & $18.4 \pm 4.6$ & $14.6 \pm 3.9$ \\
\hline NRS that reduced to less than 3 (n) & & $146(96.1 \%)$ & & $122(81.3 \%)^{*}$ \\
\hline Satisfied patients (n) & & $130(85.5 \%)$ & & $112(74.7 \%)^{*}$ \\
\hline
\end{tabular}

access to knowledge related to treatment, ensured that patients knew how to seek help, and ensured that they received a timely response when suffering severe pain or adverse drug reactions.

Our results indicated no significant difference regarding pain intensity (NRS) between the two groups at $72 \mathrm{~h}$; however, the rate of NRS that reduced to less than 3 points in $24 \mathrm{~h}$ was significantly higher in the Wechat group than in the control group. Patients' satisfaction with pain management and pain intensity was significantly higher in the Wechat group than in the control group. There were no significant differences between the two groups regarding the other findings at $72 \mathrm{~h}$, including cancer related quality of life (QOL), anxiety (GAD7 ), depression (PHQ-9) and sleep (PSQI).

The frequent occurrence of unwanted side effects may be a barrier to optimal dosing and patients' compliance, especially for patients at home. It has been suggested that the goal of the opioid titration treatment of cancerrelated pain in outpatients is to find a favorable balance between pain control and side effects [22, 23]. However, no significant scores were found for two groups for constipation, nausea, vomiting, dizziness, somnolence, pruritus, loss of consciousness, and death. The majority of the adverse effects were mild to moderate in severity,

Table 3 Treatment-related adverse events reported during the procedure. ${ }^{*} p<0.05$

\begin{tabular}{lll}
\hline Outcome measures & Wechat group $(n=152)$ & Control group $(n=150)$ \\
\hline Constipation & $20(13.2 \%)$ & $22(14.5 \%)$ \\
Nausea & $10(6.6 \%)$ & $12(8.0 \%)$ \\
Vomiting & $7(4.6 \%)$ & $9(6.0 \%)$ \\
Dizziness & $5(3.3 \%)$ & $8(5.3 \%)$ \\
Somnolence & 0 & $1(0.7 \%)$ \\
Pruritus & $1(0.7 \%)$ & 0 \\
Loss of consciousness & 0 & 0 \\
Death & 0 & 0 \\
\hline
\end{tabular}

with similar profiles for the two groups. This may be mainly because our research mainly focuses on the incidence of adverse events, while the treatment of these adverse reactions needs a certain period of time. In the future, we need to pay attention to the effect of wechat education on the treatment of adverse reactions. Taken together, these results suggest the beneficial effect of reinforced education using Wechat in the management of the opioid titration treatment of cancer-related pain in outpatients.

Compared to care as usual, reinforced education using Wechat result in better pain control and better patients' satisfaction, contributing to improve cancer pain in outpatients. The intervention could be used for other cancer-related health problems or pain problems in other chronic disease populations [8, 21, 24]. Patients require assistance to easily access information on pain and pain medication, and on when and how to get help [25, 26]. Patients also need to be able to recognize and monitor pain and adverse events to gain insight into their own situation and feedback about how they are doing. In the current period of virus epidemic, Wechat may also optimize the pain management and prevents crossinfections among medical workers and patients, which may help to alleviate shortages in the medical workforce to fight COVID-19 on the front line [27].

This study has some limitations. As this study mainly evaluated the effect of Wechat-based reinforced education on opioid titration, the follow-up time was only 3 days. In the future, evaluating the long-term help of Wechat for cancer pain management should be considered. In addition, although we try to obtain patient's information through data acquisition and analysis in order to obtain better clinical protocols, it is impossible to do big data analysis due to the limitation of the number of patients. However, this may be a good research direction in the future. Better programs should be developed to apply to a larger number of people, so as to do big data analysis and artificial intelligence analysis to obtain the 
optimal diagnosis and treatment automatically in the future, which will better serve for public health.

\section{Conclusions}

Our results indicated that patients receiving instructions delivered by Wechat had a relatively higher quality of opioid titration treatment and better satisfaction. To provide long-term support for a wider population of cancer patients with pain, clinicians should integrate evidence-based activities to support reinforced education in routine clinical practice.

\section{Abbreviations}

NRS: Numerical rating scale; QOL: Cancer-related quality of life; GAD7: Generalized anxiety disorder-7; PHQ-9: Patient health questionnaire-9; PSQI: Pittsburgh sleep quality index; WHO: World Health Organization; NCCN: National Comprehensive Cancer Network

\section{Acknowledgements}

We thanks to the editorial department of Journal of Zhejiang University for polishing this article.

\section{Authors' contributions}

ZYP and XMF conceived and designed the study. ZYP, LL and YC performed the study. ZYP and ZYF analyzed the data. ZYP wrote the paper and ZYF critically reviewed the manuscript. All authors have read and approved the manuscript.

\section{Funding}

This research was supported by Zhejiang health and Family Planning Commission (Grant no. 2019RC173) to Dr. Zhiyou Peng and Hangzhou Science and Technology Commission (20180533B36) to Yuan Chen. These are earmarked funds for cancer research. The funding bodies have no role in study design, data collection, data analysis, data interpretation, or manuscript preparation.

\section{Availability of data and materials}

The data used to support the findings of this study are available from the corresponding author upon request.

\section{Ethics approval and consent to participate}

The study was approved by the institutional review board of the first affiliated hospital, Zhejiang University School of Medicine, and was registered at chictr.org (ChiCTR1900021150). The written consent statement were obtained from all individual participants. Informed consent was obtained from all individual participants included in the study.

\section{Consent for publication}

Not applicable.

\section{Competing interests}

The authors declare that they have no competing of interests.

\section{Author details \\ 'Department of Pain Medicine, the First Affiliated Hospital, Zhejiang University School of Medicine, Hangzhou, China. ${ }^{2}$ Department of Anesthesiology, Yuyao people's Hospital, Ningbo, China. ${ }^{3}$ Department of Anesthesiology, Hangzhou first people's Hospital, Hangzhou, China. ${ }^{4}$ Department of Anesthesiology, the First Affiliated Hospital, Zhejiang University School of Medicine, Hangzhou, China.}

Received: 31 March 2020 Accepted: 7 August 2020

Published online: 04 September 2020

\section{References}

1. Schumacher KL, Plano Clark VL, West CM, Dodd MJ, Rabow MW, Miaskowsk C. Pain medication management processes used by oncology outpatients and family caregivers part II home and lifestyle contexts. J Pain Symptom Manag. 2014:48:784-96.

2. Ebenau A, Dijkstra B, Ter Huurne C, Hasselaar J, Vissers K, Groot M. Palliative care for people with substance use disorder and multiple problems: a qualitative study on experiences of patients and proxies. BMC Palliat Care. 2019:18(1):56.

3. Payne S, Turner M, Seamark D, Thomas C, Brearley S, Wang X, Blake S, Milligan C. Managing end of life medications at home-accounts of bereaved family carers: a qualitative interview study. BMJ Support Palliat Care. 2015;5: $181-8$.

4. ElMokhallalati Y, Mulvey MR, Bennett MI. Interventions to support selfmanagement in cancer pain. Pain Rep. 2018;3:e690.

5. Schulman-Green D, Feder S. Integrating family caregivers into palliative oncology care using the self- and family management approach. Semin Oncol Nurs. 2018:34(3):252-63.

6. van Ryn M, Sanders S, Kahn K, van Houtven C, Griffin JM, Martin M, Atienza AA, Phelan S, Finstad D, Rowland J. Objective burden, resources, and other stressors among informal cancer caregivers: a hidden quality issue? Psychooncology. 2011;20:44-52.

7. McCorkle R, Ercolano E, Lazenby M, Schulman-Green D, Schilling LS, Lorig K, Wagner EH. Self-management: enabling and empowering patients living with cancer as a chronic illness. CA Cancer J Clin. 2011;61(1):50-62.

8. Latter S, Hopkinson JB, Richardson A, Hughes JA, Lowson E, Edwards D. How can we help family carers manage pain medicines for patients with advanced cancer? A systematic review of intervention studies. BMJ Support Palliat Care. 2016;6(3):263-75.

9. Thomas ML, Elliott JE, Rao SM, Fahey KF, Paul SM, Miaskowski C. A randomized, clinical trial of education or motivational-interviewing-based coaching compared to usual care to improve cancer pain management. Oncol Nurs Forum. 2012:39:39-49.

10. Oldenmenger WH, Sillevis Smitt PA, van Dooren S, Stoter G, van der Rijt CC. A systematic review on barriers hindering adequate cancer pain management and interventions to reduce them: a critical appraisal. Eur J Cancer. 2009:45(8):1370-80.

11. St Louis C, Zorlu G. Can twitter predict disease outbreaks? BMJ. 2012;344: 2353

12. Davies AN, Dickman A, Reid C, et al. The management of cancer-related breakthrough pain: recommendations of a task group of the Science Committee of the Association for Palliative Medicine of Great Britain and Ireland. Eur J Pain. 2009:13:331-8.

13. Kang $X$, Zhao L, Leung F, Luo H, Wang L, Wu J, Guo X, Wang X, Zhang L, Hui N, Tao Q, Jia H, Liu Z, Chen Z, Liu J, Wu K, Fan D, Pan Y, Guo X. Delivery of instructions via Mobile Social media app increases quality of bowel preparation. Clin Gastroenterol Hepatol. 2016;14(429-35):e3.

14. Lester P, Remolana R, Sandhu S, Scott J. Road map for opioid Management in the Inpatient Setting: a structured approach to opioid selection and titration. MedEdPORTAL. 2016:12:10470

15. Pan H, Shen P, Shu Q, Lu L, Qian S, Zhou Y, Han F, Guo Q, Yang Z, Pan J, Xu Q, Zhang P. Wang K. Efficacy and safety of sustained-release oxycodone compared with immediate-release morphine for pain titration in cancer patients: a multicenter, open-label, randomized controlled trial (SOCIAL). Medicine (Baltimore). 2019;98:e15505

16. Parkerson HA, Thibodeau MA, Brandt CP, Zvolensky MJ, Asmundson GJ. Cultural-based biases of the GAD-7. J Anxiety Disord. 2015;31:38-42.

17. Hyphantis T, Kotsis K, Kroenke K, Paika V, Constantopoulos S, Drosos AA Carvalho AF, Guthrie E. Lower PHQ-9 cut-point accurately diagnosed depression in people with long-term conditions attending the accident and emergency department. J Affect Disord. 2015;176:155-63.

18. Jiang Y, Tang Y-R, Xie C, Yu T, Xiong W-J, Lin L. Influence of sleep disorders on somatic symptoms, mental health, and quality of life in patients with chronic constipation. Medicine (Baltimore). 2017;96(7):e6093.

19. Bee $P$, Barnes $P$, Luker K. A systematic review of informal caregivers' needs in providing home-based end-of-life care to people with cancer. J Clin Nurs. 2008:18:1379-93.21.

20. Koller A, Miaskowski C, De Geest S, Opitz O, Spichiger E. Supporting selfmanagement of pain in cancer patients: methods and lessons learned from a randomized controlled pilot study. Eur J Oncol Nurs. 2013;17:1-8.

21. Hochstenbach LM, Courtens AM, Zwakhalen SM, van Kleef M, de Witte LP. Self-management support intervention to control cancer pain in the outpatient setting: a randomized controlled trial study protocol. BMC Cancer. 2015;15:416. 
22. Hartung DM, Middleton L, Haxby DG, Koder M, Ketchum KL, Chou R. Rates of adverse events of long-acting opioids in a state Medicaid program. Ann Pharmacother. 2007:41:921-8.

23. Webster L. Efficacy and safety of dual-opioid therapy in acute pain. Pain Med. 2012;13(suppl 1):S12-20

24. Valenta S, Spirig R, Miaskowski C, Zaugg K, Spichiger E. Testing a pain selfmanagement intervention by exploring reduction of analgesics' side effects in cancer outpatients and the involvement of family caregivers: a study protocol (PEINCA-FAM). BMC Nurs. 2018;17:54

25. Lee YJ, Kim ES, Choi JH, Lee KI, Park KS, Cho KB, Jang BK, Chung WJ, Hwang JS. Impact of reinforced education by telephone and short message service on the quality of bowel preparation: a randomized controlled study. Endoscopy. 2015:47:1018-27.

26. Liu X, Luo H, Zhang L, Leung FW, Liu Z, Wang X, Huang R, Hui N, Wu K, Fan D, Pan Y, Guo X. Telephone-based re-education on the day before colonoscopy improves the quality of bowel preparation and the polyp detection rate: a prospective, colonoscopist-blinded, randomised, controlled study. Gut. 2014;63:125-30

27. Xu H, Huang S, Qiu C, Liu S, Deng J, Jiao B, Tan X, Ai L, Xiao Y, Belliato M, Yan L. Monitoring and Management of Home-Quarantined Patients with COVID-19 using a WeChat-based telemedicine system: retrospective cohort study. J Med Internet Res. 2020;22(7):e19514.

\section{Publisher's Note}

Springer Nature remains neutral with regard to jurisdictional claims in published maps and institutional affiliations.

Ready to submit your research? Choose BMC and benefit from:

- fast, convenient online submission

- thorough peer review by experienced researchers in your field

- rapid publication on acceptance

- support for research data, including large and complex data types

- gold Open Access which fosters wider collaboration and increased citations

- maximum visibility for your research: over $100 \mathrm{M}$ website views per year

At BMC, research is always in progress.

Learn more biomedcentral.com/submissions 